\title{
Valores de fuerza prensil de mano en sujetos aparentemente sanos de la ciudad de Cúcuta, Colombia
}

\section{Hand grip strength values in apparently healthy subjects of the city of Cúcuta, Colombia \\ Valores deforçadepreensãomanualemindivíduosaparentemente sadios da cidade de Cúcuta, Colômbia}

Brian Johan Bustos-Viviescas, Lic. *

Andrés Alonso Acevedo-Mindiola, Lic.*

Rafael Enrique Lozano-Zapata, PhD. ${ }^{* *}$

\section{Resumen}

Introducción. La fuerza prensil de la mano es un parámetro fundamental por evaluar y para conocer el nivel de fuerza muscular; sin embargo, a nivel nacional son escasos los estudios que determinan la fuerza en extremidades superiores. Objetivo. Evaluar la fuerza prensil de la mano en sujetos aparentemente sanos de la ciudad de Cúcuta, Colombia. Metodología. Estudio transversal, observacional con muestreo no probabilístico de tipo intencional, con participación voluntaria de 162 hombres y 228 mujeres. Todos los resultados en la fuerza prensil de la mano fueron categorizados por sexo, década, mano dominante y mano no dominante. Para el análisis estadístico se utilizó el paquete estadístico IBM SPSS V.22 bajo un nivel de confianza del $95 \%$ y un p-valor de 0.05 y se realizaron las pruebas de normalidad de Kolmogorov-Smirnov y el análisis de varianza. Resultados. Se evidenció en los hombres en la los rangos de edad de 20 a 29 años el pico más alto de fuerza; no obstante, a partir de allí, a medida que aumenta la edad la fuerza prensil de la mano disminuye. En mujeres se incrementa paulatinamente hasta el rango de edad 30 a 39 años. En el análisis de varianza $(\mathrm{p}>0.05)$ se demuestra que no existe diferencia significativa entre la fuerza prensil de mano dominante y no dominante en ningún intervalo de década para hombres y mujeres. Conclusiones. La fuerza prensil de mano aumenta hasta el rango de edad de 30 a 39 en ambos sexos. Además, no hay diferencias significativas entre la fuerza prensil de la mano dominante y no dominante por década. [Bustos-Viviescas BL, AcevedoMindiola AA, Lozano-Zapata RE. Valores de fuerza prensil de mano en sujetos aparentemente sanos de la ciudad de Cúcuta, Colombia. MedUNAB. 2019;21(3):363-377. doi:10.29375/01237047.2791]

Palabras Clave: Personas con Discapacidad; Mano; Fuerza

\footnotetext{
* Licenciado en Educación Básica con Énfasis en Educación Física, Recreación y Deportes de la Universidad de Pamplona. Integrante del Semillero de Investigación de las Ciencias de la Actividad Física y el Deporte (SICAFD), Cúcuta, Norte de Santander, Colombia.

** Doctor en Ciencias de la Actividad Física y el Deporte. Docente de planta de la Universidad de Pamplona. Tutor del Semillero de Investigación de las Ciencias de la Actividad Física y el Deporte (SICAFD), Cúcuta, Norte de Santander, Colombia.
}

Correspondencia: Brian Johan Bustos Viviescas. Dirección postal: Departamento de Educación Física, Unipamplona Sede Villa del Rosario. Celular: 3173074022 E-mail: brian.bustos@unipamplona.edu.co 
de la Mano; Fuerza Muscular; Dinamómetro de Fuerza Muscular; Valores de Referencia.

\begin{abstract}
Introduction. Hand grip strength is a fundamental parameter to be assessed and to get to know muscle strength. However, at the national level, few studies determine the strength of the upper limbs. Objective. Assess hand grip strength in apparently healthy subjects of the city of Cúcuta, Colombia. Methodology. Cross-sectional observational study with intentional non-probabilistic sampling, and the voluntary participation of 162 men and 228 women. All of the hand grip strength results were categorized by sex, decade, dominant hand and non-dominant hand. For the statistical analysis, the IBM SPSS Statistics V.22 software was used with a confidence level of $95 \%$ and a p-value of 0.05 , and Kolmogorov-Smirnov normality tests and analysis of variance were conducted. Results. It was demonstrated that men in the age range of 20 to 29 years had the highest peak of strength. However, after that, as age increased, hand grip strength decreased. In women, it increased gradually up to the age range of 30 to 39 years. In the analysis of variance $(\mathrm{p}>0.05)$, it was demonstrated that there is no significant difference between the hand grip strength of the dominant hand and the non-dominant had at any of the ten-year age intervals for men and women. Conclusions. Hand grip strength increased up to the age range of 30 to 39 years in both sexes. In addition, there are no significant differences between the hand grip strength of the dominant hand and the non-dominant hand by ten-year age interval. [BustosViviescas BL, Acevedo-Mindiola AA, Lozano-Zapata RE. Hand Grip Strength Values in Apparently Healthy Subjects of the City of Cúcuta, Colombia. MedUNAB. 2019;21(3):363377. doi:10.29375/01237047.2791]
\end{abstract}

Keywords: Disabled; Hand; Hand Strength; Muscle Strength; Muscle Strength Dynamometer; Reference Values.

\section{Resumo}

Introdução. A força de preensão manual é um parâmetro fundamental para avaliar e conhecer o nível de força muscular; no entanto, em todo o país existem poucos estudos que falem sobre a força nas extremidades superiores. Objetivo. Avaliar a força de preensão manual em indivíduos aparentemente sadios da cidade de Cúcuta, Colômbia. Métodos. Estudo observacional, de caráter transversal com amostragem não probabilística do tipo intencional, com a participação voluntária de 162 homens e 228 mulheres. Todos os resultados em força de preensão manual foram categorizados por sexo, faixas etárias, mão dominante e não dominante. Para a análise estatística utilizou-se o IBM
SPSS Statistics 22.0, com nível de confiança de $95 \%$ e um p-valor de 0.05 e foram utilizados testes de normalidade de Kolmogorov-Smirnov e análise de variância. Resultados. Foi evidenciado um pico maior da força em homens nas faixas etárias de 20 a 29 anos; no entanto, há um declínio da força manual à medida que a idade aumenta. Nas mulheres, aumenta gradualmente até a faixa etária de 30 a 39 anos de idade. A análise de variância $(\mathrm{p}>0.05)$ mostra que não há diferença significativa entre a força de preensão na mão dominante e não dominante em qualquer intervalo de faixa etária para homens e mulheres. Conclusão. A força de preensão de mão aumenta até a faixa etária de 30 a 39 em ambos os sexos. Além disso, não há diferenças significativas entre a força de preensão na mão dominante e na não dominante nas faixas etárias. [Bustos-Viviescas $B L$, Acevedo-Mindiola AA, Lozano-Zapata RE. Valores de força de preensão manual em individuos aparentemente sadios da cidade de Cúcuta, Colômbia. MedUNAB. 2019;21(3):363377. doi:10.29375/01237047.2791]

Palavras-chave: Pessoas com Deficiência; Mãos; Força da Mão; Força Muscular; Dinamômetro de Força Muscular; Valores de Referência.

\section{Introducción}

La fuerza de agarre es una prueba que evalúa la funcionalidad de la mano, por ello se hace necesario la medición de la Fuerza Prensil de Mano (FPM) o fuerza de agarre, por su gran importancia clínica en la evaluación de la extremidad superior (1), puesto que se evidencia una estrecha relación entre dinamometría manual con la integridad funcional de la extremidad superior (2) y la habilidad para realizar actividades de la vida diaria (3). Otro rasgo de esta técnica es que permite obtener valores que se pueden utilizar para determinar el resultado de un manejo médico ortopédico o quirúrgico de la patología de la mano o el resultado de tratamientos de rehabilitación que establezcan cuándo un sujeto está en condiciones para volver a sus actividades normales (1).

La fuerza de la mano medida por dinamometría manual puede estimar el desarrollo biológico de la persona $y$, en consecuencia, sirve como referencia para el seguimiento en los procesos de rehabilitación (4). A su vez, es un buen indicador de salud, ya que nos predice el estado de salud presente y futuro (5). De esta manera, en una investigación desarrollada con universitarios colombianos se establece que la valoración de la fuerza de agarre mediante dinamometría manual se considera actualmente como un indicador del estado nutricional 
y un marcador temprano en la morbimortalidad de la enfermedad cardiometabólica (6), por lo que es necesario la existencia de valores normales para la interpretación de los datos de la evaluación de un paciente (1), debido a que en los programas de prevención y promoción de la salud es fundamental evaluar este parámetro para establecer el estado de salud de la población a intervenir.

Estudios epidemiológicos a nivel nacional destacan la importancia de evaluar la fuerza prensil de la mano para el proceso de diagnóstico de las alteraciones de la mano, así como para establecer deficiencias del miembro superior (7-8), para ello se requiere del desarrollo de datos normativos que permitan interpretar adecuadamente los datos obtenidos durante la medición; no obstante, los valores estándar deben ser acordes a las características de una población específica (9).

Teniendo en cuenta lo anterior, los profesionales de la salud y del deporte deben utilizar la dinamometría manual como prueba física debido a que permite identificar el estado de salud del sujeto y, siendo más precisos, porque tiene aplicabilidad en el campo de la salud con el propósito de medir los niveles de fuerza en mano de los sujetos sanos o pacientes clínicos; no obstante, las estrategias de medición de la FPM se basan principalmente en escalas extranjeras. Estas pierden su verdadero valor cuando son utilizadas en la población colombiana, teniendo en cuenta que los sujetos distan mucho en las características somatotípicas, sociodemográficas y nutricionales (10).

Por tal motivo, es necesario evaluar la FPM y compararla con valores de referencia para la misma población con el propósito de identificar si los niveles de fuerza prensil del sujeto son adecuados para la edad. Tomar tales acciones posibilita desarrollar objetivamente los programas de prevención y promoción de la salud que emplean esta variable.

En consecuencia, se dificulta la tarea de evaluar los avances en el proceso de rehabilitación de la capacidad funcional manual de sujetos con determinadas alteraciones, y de aquellas poblaciones en las cuales se quiera establecer el estado de salud, debido a que no se cuentan con parámetros de normalidad en la fuerza de agarre de sujetos sanos de diferentes rangos de edad y género con desarrollo psicomotor normal de la ciudad de San José de Cúcuta. Por otra parte, cabe resaltar que los escasos estudios desarrollados que evaluaron la FPM específicamente en la ciudad de Cúcuta han evidenciado que esta variable tiene una relación significativa con la presión arterial sistólica y diastólica en mujeres, mientras que en los hombres solamente con la presión arterial diastólica (11); así mismo, el porcentaje de grasa corporal está relacionado con la fuerza prensil de ambas manos en hombres, y en el caso de las mujeres con la mano dominante (12).

Por lo tanto, el objetivo del estudio fue evaluar la FPM en sujetos aparentemente sanos de la ciudad de Cúcuta, Colombia, para obtener valores de referencia que puedan ser utilizados en la práctica clínica por profesionales de la salud y del deporte en sus evaluaciones sobre el estado de salud o el grado de recuperación de la extremidad superior.

\section{Metodología}

Este estudio es producto del proyecto denominado "Los beneficios de la actividad física, como factor fundamental del buen estado físico y salud de las personas que asisten los días domingos a la ciclovía del malecón de la ciudad de Cúcuta" (13).

El estudio es transversal, observacional, con muestreo no probabilístico de tipo intencional (selección de la muestra de acuerdo con unos criterios establecidos por el investigador) (14). Este muestreo se realizó con 390 personas que participaron voluntariamente. La distribución de la muestra es de 162 hombres y 228 mujeres. Para determinar el tamaño de esta se aplicó la siguiente fórmula debido a que no se conoce con precisión el tamaño de la población. Se obtuvo como resultados un tamaño de muestra de 384.16 sujetos.

Fórmula de cálculo:

Donde:

$n=$

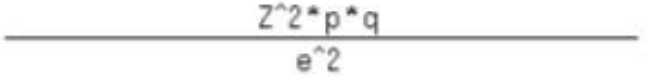

Z: es el nivel de confianza (el valor de confianza fue de $95 \%$ equivalente a 1.96)

p: es el porcentaje de la población que tiene el atributo deseado*

q: es el porcentaje de la población que no tiene el atributo deseado

* cuando no hay indicación de la población que posee o no el atributo, se asume $50 \%$ para p y $50 \%$ para q. 
e: es el error de estimación máximo aceptado ( $5 \%$ fue el error máximo para un nivel de confianza del $95 \%$ ).

Entre los criterios de inclusión para participar en el estudio se encuentran los siguientes: vivir en la Ciudad de San José de Cúcuta, participación voluntaria y no presentar dolor reciente en manos y/o brazos. Por otro lado, los criterios de exclusión fueron los siguientes: haber entrenado la fuerza en los últimos tres meses (fisicoculturismo, deportistas de alto rendimiento, entre otros), estar trabajando o realizando actividades de uso continuo de extremidades superiores (constructor, agricultor, entre otros), ser ambidiestro y presentar alguna patología que pudiera afectar la fuerza muscular de las extremidades superiores y/o tener sensación de molestia o dolor durante la evaluación.

Para la toma de datos se utilizaron los siguientes instrumentos:

Báscula TANITA BC-730: utilizada para obtener el peso corporal de cada participante, precisión de $100 \mathrm{~g}$. Fabricante: TANITA Corporation of América Inc., USA.

Tallímetro de pared seca 206: empleado para medir la estatura $(0-220 \mathrm{~cm})$, precisión $1 \mathrm{~mm}$. Fabricante: Seca gmbh, Alemania.

Dinamómetro digital Camry: empleado para medir la fuerza prensil (fuerza de agarre de hasta $200 \mathrm{lb} / 90 \mathrm{~kg}$, división de $0.2 \mathrm{lb} / 100 \mathrm{~g}$ ). Fabricante: GENERAL ASDE S. A., España.

Los datos fueron recolectados en la misma hora del día (7:00-9:00 am) en el periodo comprendido entre julio y noviembre de 2016. Los datos de la talla y el peso fueron tomados a los participantes con ropa ligera y sin calzado; se tomó un registro de cuál de las dos manos usan los participantes principalmente en sus actividades diarias con el fin de clasificar los resultados de la fuerza de agarre en mano dominante y mano no dominante; posteriormente se realizó la dinamometría en ambas manos con un descanso de 3 minutos aproximadamente, y se repetía nuevamente la misma para utilizar el valor más alto de cada mano, igualmente el dinamómetro se ajustaba de acuerdo al tamaño de la mano de cada participante. Hay que destacar que los equipos se calibraban periódicamente para evitar sesgos de medición en la investigación.

Durante la evaluación de la fuerza prensil de la mano o fuerza de agarre, el participante estaba de pie, en posición firme, con el brazo totalmente extendido y paralelo al tronco, se indicó verbalmente al participante que aplicara su fuerza máxima de manera continua hasta que el dinamómetro no registrara incremento durante unos segundos, durante la prueba el participante no podía levantar la mano ni realizar ningún movimiento compensatorio que alterara la posición inicial.

Esta investigación se realizó de acuerdo con los principios éticos a nivel internacional para las investigaciones médicas en seres humanos expuestos en la Declaración de Helsinki de la Asociación Médica Mundial (15) y los estándares éticos establecidos para investigaciones en ciencias del deporte y del ejercicio (16). De otra parte, se tuvo en cuenta las normas científicas, técnicas y administrativas para la investigación en salud indicados en la resolución $\mathrm{N}^{\mathrm{o}}$ 008430 de 1993 del Ministerio de Salud de Colombia y se clasificó este estudio en una categoría de riesgo mínimo, según lo establecido en su artículo 11, numeral b (17). Así mismo, esta investigación cuenta con el aval del comité de ética e impacto ambiental mediante el Acta $\mathrm{N}^{\circ} 002$ del 16 de abril del 2018.

Para la tabulación y análisis de los resultados obtenidos se utilizó el paquete estadístico IBM SPSS V.22; en este paquete estadístico se estableció un nivel de confianza del 95\% y un p-valor de 0.05 , se aplicaron las pruebas de normalidad (KolmogorovSmirnov) y análisis de varianza (ANOVA). De igual modo, se calcularon los percentiles 5, 10, 25, 50, 75,90 y 95 para cada sexo y ajustado a intervalos de décadas para posibilitar la comparación con otros grupos que se encontraran en los mismos rangos de edad. A partir de esto se utilizaron los puntos de corte propuestos para el diagnóstico de la aptitud física (18): Deficiente $(<\mathrm{P} 10)$, Mala (P10 a P25), Regular (P25 a P50), Bueno (P50 a P75), Muy bueno (P75 a P90) y Excelente (>P90).

Todos los participantes firmaron un consentimiento informado donde estaban detalladas todas las mediciones y evaluaciones a realizar. Así mismo, se cedieron los datos de los resultados obtenidos con fines de investigación siempre y cuando se respete la confidencialidad del participante.

Con los participantes menores de edad que aceptaron participar en el estudio voluntariamente, se realizó el correspondiente consentimiento informado el cual fue firmado por sus padres, madres o tutores legales, y el debido asentimiento del participante. 


\section{Resultados}

\section{Características generales}

La muestra del estudio está constituida por 162 hombres que representan el $41.53 \%$ de la muestra. La edad promedio de los hombres es de $34.40+16.68$ años. Además, se destaca que los hombres con mayor peso corporal se encuentran en los rangos de edad de 30 a 39 $(79.91+13.44 \mathrm{~kg})$ y 40 a $49(78.55+13.42 \mathrm{~kg})$ (Tabla 1).

Con respecto a las mujeres, participaron 228 que representa el $58.46 \%$ de la muestra, con un promedio de edad de $30.38+15.86$ años. De otra parte, se aprecia que en el rango de edad de 40 a 49 años $(64.89+7.91$ $\mathrm{kg}), 50$ a 59 años $(66.29+9.63 \mathrm{~kg})$ y 60 a 69 años $(64.53+6.20 \mathrm{~kg})$ hay un mayor peso corporal en comparación con los demás rangos de edad (Tabla 2).

\section{Fuerza de agarre en hombres y mujeres}

A continuación, se puede apreciar los valores de fuerza de agarre en mano dominante y no dominante de hombres por rangos de edad (Tabla 3). Se evidencia que hasta el rango de edad de 30 a 39 años la fuerza de agarre en mano dominante y no dominante aumenta; luego, a mayor edad los valores de fuerza van disminuyendo, obteniendo así que los hombres del rango de edad de los 60 a 69 años tienen un promedio de fuerza en la mano dominante de $30.61+7.35 \mathrm{~kg}$ y en la mano no dominante de $27.60+6.02 \mathrm{~kg}$. Cabe mencionar que no hay diferencia significativa entre las medias de fuerza de agarre en la mano dominante y la mano no dominante respecto al grupo de edad en hombres $(\mathrm{P}>0.05)$.

De igual manera, en mujeres se observa que va aumentando progresivamente la fuerza prensil de mano desde el rango de edad de 10 a 19 años (fuerza en mano dominante $23.01+5.09 \mathrm{~kg}$, fuerza en mano no dominante $22.44+5.07 \mathrm{~kg}$ ) hasta el rango de edad de 30 a 39 años (fuerza en mano dominante $27.87+6.25$ $\mathrm{kg}$, fuerza en mano no dominante $24.46+6.47 \mathrm{~kg}$ ), luego, del mismo modo que en hombres, la capacidad de generar fuerza en ambas manos va disminuyendo, destacando que en el rango de edad de 50 a 59 años se encontraron valores inferiores de fuerza en mano dominante $(20.76+5.64 \mathrm{~kg})$ y mano no dominante $(20.90+5.92 \mathrm{~kg})$ en comparación con los demás rangos de edad (Tabla 4). Por otro lado, de igual forma que en los hombres, no hubo diferencias significativas entre las medias de fuerza prensil de la mano dominante y no dominante con respecto al grupo de edad $(\mathrm{P}>0.05)$.

\section{Normalidad de los datos}

Se encontró que solamente en los hombres existe una distribución normal en la fuerza de agarre para ambas manos ( $p>0.05)$, mientras que las mujeres obtuvieron una distribución no normal para ambas manos $(\mathrm{p}<0.05)$. Así mismo, en las (Figura 1) y (Figura 2)

Tabla 1. Características generales de los hombres

\begin{tabular}{|c|c|c|c|c|c|}
\hline \multicolumn{6}{|c|}{ Hombres } \\
\hline Décadas & n & Edad & Peso (kg) & Talla (cm) & $\begin{array}{c}\text { IMC (kg/ } \\
\text { m2) }\end{array}$ \\
\hline 10 a 19 & 38 & $16.91 \pm 3.20$ & $65.01 \pm 12.75$ & $171.98 \pm 9.76$ & $21.47 \pm 3.62$ \\
\hline 20 a 29 & 48 & $23.58 \pm 2.34$ & $71.45 \pm 11.74$ & $171.92 \pm 7.20$ & $24.28 \pm 3.23$ \\
\hline 30 a 39 & 24 & $33.72 \pm 2.84$ & $79.91 \pm 13.44$ & $173.08 \pm 7.94$ & $25.45 \pm 4.76$ \\
\hline 40 a 49 & 15 & $45.40 \pm 3.11$ & $78.55 \pm 13.42$ & $170.27 \pm 4.82$ & $27.00 \pm 3.99$ \\
\hline 50 a 59 & 25 & $54.50 \pm 3.32$ & $75.33 \pm 15.50$ & $167.14 \pm 6.58$ & $26.28 \pm 4.47$ \\
\hline 60 a 69 & 12 & $63.44 \pm 3.01$ & $73.88 \pm 15.55$ & $167.25 \pm 6.24$ & $26.18 \pm 5.37$ \\
\hline Total & 162 & $34.40 \pm 16.68$ & $72.73 \pm 13.91$ & $170.79 \pm 7.80$ & $24.53 \pm 4.38$ \\
\hline
\end{tabular}

Fuente: Elaborado por los autores. 
Tabla 2. Características generales de las mujeres

\begin{tabular}{|c|c|c|c|c|c|}
\hline \multicolumn{7}{|c|}{ Mujeres } \\
\hline Décadas & n & Edad & Peso (kg) & Talla (cm) & IMC (kg/m²) \\
\hline 10 a 19 & 82 & $15.66 \pm 1.97$ & $52.53 \pm 8.66$ & $162.21 \pm 7.83$ & $20.49 \pm 2.95$ \\
\hline 20 a 29 & 64 & $23.87 \pm 2.69$ & $58.84 \pm 9.13$ & $161.08 \pm 5.78$ & $22.73 \pm 3.60$ \\
\hline 30 a 39 & 19 & $33.30 \pm 3.14$ & $60.72 \pm 11.46$ & $159.96 \pm 6.72$ & $24.35 \pm 4.83$ \\
\hline 40 a 49 & 29 & $45.12 \pm 3.00$ & $64.89 \pm 7.91$ & $157.08 \pm 4.64$ & $22.82 \pm 2.98$ \\
\hline 50 a 59 & 24 & $53.48 \pm 3.08$ & $66.29 \pm 9.63$ & $158.63 \pm 8.50$ & $25.29 \pm 3.76$ \\
\hline 60 a 69 & 10 & $62.67 \pm 1.97$ & $64.53 \pm 6.20$ & $158.83 \pm 5.52$ & $25.73 \pm 1.95$ \\
\hline Total & 228 & $30.38 \pm 15.86$ & $58.78 \pm 10.42$ & $160.48 \pm 6.96$ & $23.04 \pm 4.03$ \\
\hline
\end{tabular}

Fuente: Elaborado por los autores.

Tabla 3. Valores de fuerza de agarre en los hombres

\begin{tabular}{|c|c|c|c|c|}
\hline \multicolumn{5}{|c|}{ Hombres } \\
\hline Décadas & n & $\begin{array}{c}\text { Mano Dominante } \\
(\mathbf{k g})\end{array}$ & $\begin{array}{c}\text { Mano no } \\
\text { Dominante (kg) }\end{array}$ & $\begin{array}{c}\text { Análisis de varianza } \\
\text { (ANOVA) }\end{array}$ \\
\hline 10 a 19 & 38 & $35.11 \pm 10.09$ & $33.54 \pm 9.65$ & 0.66 \\
\hline 20 a 29 & 48 & $40.34 \pm 7.39$ & $39.63 \pm 7.24$ & 0.63 \\
\hline 30 a 39 & 24 & $39.42 \pm 12.54$ & $39.26 \pm 12.11$ & 0.96 \\
\hline 40 a 49 & 15 & $38.05 \pm 8.61$ & $35.57 \pm 9.46$ & 0.46 \\
\hline 50 a 59 & 25 & $32.57 \pm 8.32$ & $31.96 \pm 9.06$ & 0.82 \\
\hline 60 a 69 & 12 & $30.61 \pm 7.35$ & $27.60 \pm 6.02$ & 0.23 \\
\hline Total & 162 & $36.71 \pm 9.61$ & $35.71 \pm 9.74$ & 0.32 \\
\hline Nota: ${ }^{*}$ p $<0.05$ (existe diferencia entre las medias) & \\
\hline
\end{tabular}

Fuente: Elaborado por los autores. 
Tabla 4. Valores de fuerza de agarre en las mujeres

\begin{tabular}{|c|c|c|c|c|}
\hline \multicolumn{5}{|c|}{ Mujeres } \\
\hline Décadas & n & $\begin{array}{c}\text { Mano Dominante } \\
(\mathbf{k g})\end{array}$ & $\begin{array}{c}\text { Mano no } \\
\text { Dominante (kg) }\end{array}$ & $\begin{array}{c}\text { Análisis de varianza } \\
\text { (ANOVA) }\end{array}$ \\
\hline 10 a 19 & 82 & $23.01 \pm 5.09$ & $22.44 \pm 5.07$ & 0.49 \\
\hline 20 a 29 & 64 & $26.38 \pm 7.62$ & $25.06 \pm 6.28$ & 0.20 \\
\hline 30 a 39 & 19 & $27.87 \pm 6.25$ & $24.46 \pm 6.47$ & 0.47 \\
\hline 40 a 49 & 29 & $24.26 \pm 5.44$ & $23.55 \pm 4.42$ & 0.62 \\
\hline 50 a 59 & 24 & $20.76 \pm 5.64$ & $20.90 \pm 5.92$ & 0.51 \\
\hline 60 a 69 & 10 & $22.20 \pm 8.65$ & $21.02 \pm 5.36$ & 0.70 \\
\hline Total & 228 & $24.37 \pm 6.87$ & $23.33 \pm 5.91$ & 0.09 \\
\hline \multicolumn{2}{|c|}{ Nota: $* \mathrm{p}<0.05$ (existe diferencia entre las medias) } \\
\hline
\end{tabular}

Fuente: Elaborado por los autores.

se destaca la distribución simétrica de los resultados con respecto a la media en los hombres. Por otra parte, en las (Figura 3) y (Figura 4) es notable que existe una distribución asimétrica positiva, puesto que la mayoría de los datos están a la derecha de la media de la fuerza de agarre en las mujeres.

\section{Percentiles}

Los valores de la fuerza de agarre en la mano dominante y no dominante clasificados por sexo y rangos de edad se muestran en la (Tabla 5) y (Tabla 6)

\section{Puntos de corte}

A continuación, en la (Tabla 7) y (Tabla 8) se presentan los baremos correspondientes a los puntos de corte de acuerdo a los percentiles para las categorías deficiente, mala, regular, bueno, muy bueno y excelente por sexo y edad. En relación con los baremos y valores obtenidos en este estudio del promedio de la fuerza prensil en mano dominante y no dominante, se evidencia que tanto hombres y mujeres se encuentran en un nivel bueno de fuerza prensil de mano. Sin embargo, los hombres en la década de 4049 años se clasifican con una fuerza prensil en ambas manos de regular. Asimismo, las mujeres de la década 30-39 años se encuentran en una escala de regular.

\section{Discusión}

El objetivo de este estudio fue evaluar la fuerza prensil de la mano en sujetos aparentemente sanos de la ciudad de Cúcuta, Colombia. Entre los principales hallazgos se encontró que en hombres después del rango de edad de 30 a 39 años, a medida que aumenta la edad, la fuerza prensil en ambas manos va disminuyendo progresivamente. En conclusión, cabe resaltar que en hombres de la década de 20 a 29 años y de 30 a 39 años los valores son superiores, y en la década de 60 a 69 años los valores más bajos. En cuanto a las mujeres, va incrementándose desde la década de 10 a 19 años hasta la década de 30 a 39 años; a partir de las décadas posteriores la capacidad evaluada va descendiendo paulatinamente. Adicionalmente, se evidenció en el análisis estadístico que no existen diferencias significativas entre la mano dominante y no dominante, es decir que los valores de fuerza prensil en ambas manos son muy similares en hombres y mujeres.

Con respecto a lo anterior, en esta investigación se distribuyeron los resultados obtenidos en la fuerza de agarre de acuerdo con la edad y se pudo evidenciar 


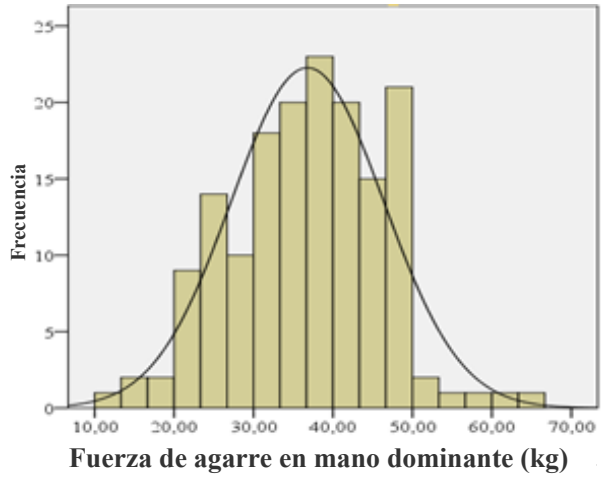

Figura 1. Distribución de la fuerza de agarre en mano dominante de los hombres

Fuente: Elaborado por los autores.

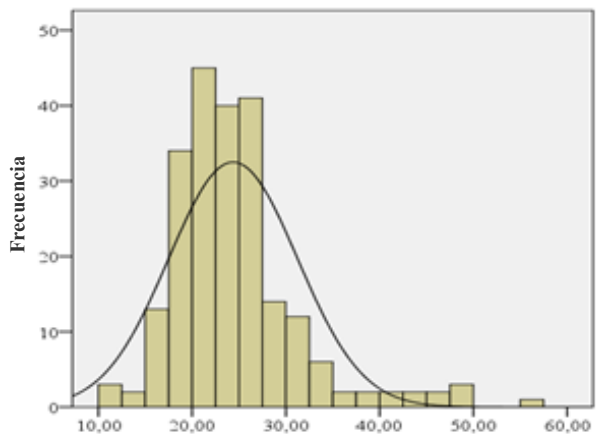

Fuerza de agarre en mano dominante $(\mathrm{kg})$

Figura 3. Distribución de la fuerza de agarre en mano dominante de las mujeres

Fuente: Elaborado por los autores.

que en todas las décadas los hombres eran más fuertes que las mujeres en ambas manos. Igualmente, otros estudios han contrastado que los hombres son más fuertes que las mujeres en la fuerza de agarre (19-25). Esta diferencia se puede relacionar con la diferencia hormonal existente en cada sexo (26), ya que el hombre, biológicamente, tiene mayor capacidad para realizar actividades que implican mayor fuerza con respecto a la mujer (27), por ende, la fuerza de agarre es consistentemente mayor en hombres que en mujeres (28) por poseer mayor masa muscular (29).

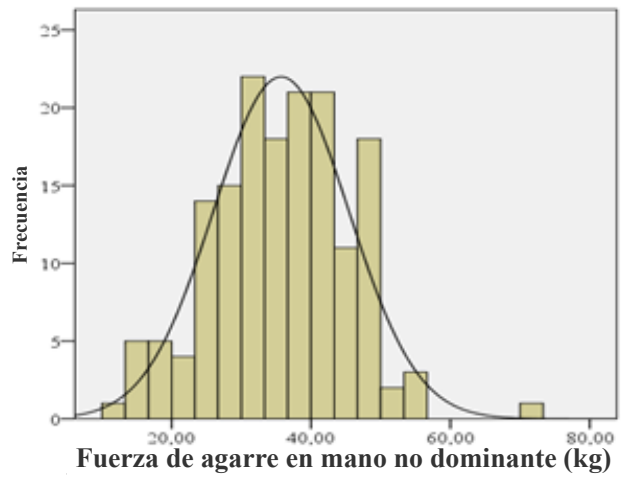

Figura 2. Distribución de la fuerza de agarre en mano no dominante de los hombres

Fuente: Elaborado por los autores.

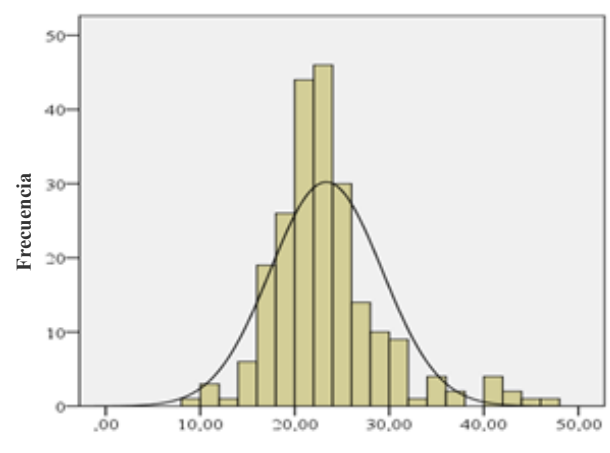

Fuerza de agarre en mano no dominante (kg)

Figura 4. Distribución de la fuerza de agarre en mano no dominante de las mujeres

Fuente: Elaborado por los autores.

De otra parte, teniendo en cuenta la fuerza prensil o de agarre y la edad, se evidencian resultados concordantes con un estudio realizado con trabajadores sanos de Manizales, Colombia. En este se evidencia que en la década de 30 a 39 años para ambos sexos fue donde se obtuvieron los valores de fuerza más altos tanto en la mano dominante y no dominante; a partir de allí declina gradualmente con la edad (8), tendencia similar a la observada en el presente estudio y en un estudio previo con individuos sanos entre 20 y 70 años, residentes en Bogotá (9). 
Tabla 5. Fuerza de agarre en la mano dominante para hombres y mujeres en percentiles

\begin{tabular}{|c|c|c|c|c|c|c|c|c|}
\hline \multicolumn{9}{|c|}{ Fuerza de agarre en la mano dominante (kg) } \\
\hline \multicolumn{9}{|c|}{ Hombres } \\
\hline Rangos de edad & $\mathrm{n}$ & p 5 & $\mathrm{p} 10$ & $\mathrm{p} 25$ & p 50 & p 75 & p 90 & p 95 \\
\hline 10 a 19 & 38 & 16.40 & 22.00 & 28.70 & 32.60 & 43.65 & 48.56 & 49.74 \\
\hline 20 a 29 & 48 & 24.95 & 30.24 & 37.07 & 40.20 & 45.85 & 48.86 & 51.06 \\
\hline 30 a 39 & 24 & 17.85 & 21.24 & 28.90 & 39.20 & 48.70 & 56.14 & 62.35 \\
\hline 40 a 49 & 15 & 24.50 & 25.52 & 30.70 & 39.00 & 46.30 & 49.36 & 49.90 \\
\hline 50 a 59 & 25 & 17.35 & 19.00 & 25.50 & 33.60 & 37.05 & 45.38 & 47.52 \\
\hline 60 a 69 & 12 & 20.20 & 21.10 & 24.70 & 30.10 & 38.50 & 41.74 & 42.70 \\
\hline \multicolumn{9}{|c|}{ Mujeres } \\
\hline Rangos de edad & $\mathrm{n}$ & p 5 & $\mathrm{p} 10$ & p 25 & p 50 & p 75 & p 90 & p 95 \\
\hline 10 a 19 & 82 & 16.66 & 18.20 & 19.90 & 22.20 & 25.30 & 29.92 & 32.26 \\
\hline 20 a 29 & 64 & 17.06 & 18.72 & 22.60 & 25.70 & 28.35 & 33.54 & 47.40 \\
\hline 30 a 39 & 19 & 17.86 & 19.49 & 24.27 & 28.45 & 31.70 & 39.33 & 42.00 \\
\hline 40 a 49 & 29 & 16.97 & 17.60 & 18.92 & 23.45 & 27.40 & 32.65 & 35.57 \\
\hline 50 a 59 & 24 & 12.66 & 15.08 & 18.70 & 20.40 & 22.70 & 29.44 & 47.22 \\
\hline 60 a 69 & 10 & 11.00 & 11.53 & 16.77 & 19.30 & 26.40 & 42.33 & 43.20 \\
\hline
\end{tabular}

Fuente: Elaborado por los autores.

Así mismo, se encontró que en una población Española la mayor fuerza de agarre se manifestaba en los grupos de edad de 31-35 años y 36-40 años (30); sin embargo, otras investigaciones indican que los niveles máximos de fuerza se alcanzan dentro de la cuarta década (31-32) y comienza el declive de la fuerza a partir de la quinta década (33), aunque en este estudio los participantes de la tercera década alcanzaron los valores más altos de fuerza de agarre y en la cuarta década se comenzó a observar un declive para ambos sexos y ambas manos, debido a que la fuerza de agarre disminuye progresivamente con la edad (34). Está pérdida de la fuerza de agarre con el avance de la edad está relacionada con la disminución de la masa muscular producto de la sarcopenia, dado que existe una pérdida de la masa muscular que declina aproximadamente un $3-8 \%$ por década a partir de la tercera década, y esta cifra incrementa cuando se llega a la sexta década (3536). Por otro lado, otros factores que intervienen en la pérdida de la fuerza muscular es la disminución del tamaño y número de fibras musculares; de igual forma, la menor activación neuronal con el avance de la edad, también disminuye en mayor medida la producción de fuerza durante acciones concéntricas (37).

Por último, en relación a la fuerza prensil y dominancia, la fuerza de agarre fue superior en los hombres con respecto a las mujeres en todas las décadas, independientemente de la dominancia. Estos mismos resultados se obtuvieron en otros estudios 
Valores de fuerza prensil de mano en sujetos aparentemente sanos de la ciudad de Cúcuta, Colombia

Tabla 6. Fuerza de agarre en la mano no dominante para hombres y mujeres en percentiles

\begin{tabular}{|c|c|c|c|c|c|c|c|c|}
\hline \multicolumn{9}{|c|}{ Fuerza de agarre en la mano no dominante (kg) } \\
\hline \multicolumn{9}{|c|}{ Hombres } \\
\hline Rangos de edad & $\mathrm{n}$ & p 5 & $\mathrm{p} 10$ & $\mathrm{p} 25$ & p 50 & $\mathrm{p} 75$ & p 90 & p 95 \\
\hline 10 a 19 & 38 & 17.31 & 21.48 & 26.05 & 32.80 & 40.55 & 49.20 & 49.72 \\
\hline 20 a 29 & 48 & 26.89 & 30.42 & 33.12 & 39.30 & 45.42 & 49.04 & 52.39 \\
\hline 30 a 39 & 24 & 17.92 & 19.50 & 31.80 & 40.30 & 47.25 & 52.46 & 67.13 \\
\hline 40 a 49 & 15 & 15.70 & 18.22 & 27.20 & 38.50 & 41.40 & 46.44 & 47.10 \\
\hline 50 a 59 & 25 & 14.51 & 17.20 & 27.30 & 30.50 & 39.25 & 46.48 & 48.81 \\
\hline 60 a 69 & 12 & 14.30 & 17.54 & 24.30 & 27.20 & 33.70 & 35.70 & 37.20 \\
\hline \multicolumn{9}{|c|}{ Mujeres } \\
\hline Rangos de edad & $\mathrm{n}$ & p 5 & p 10 & $\mathrm{p} 25$ & p 50 & p 75 & p 90 & p 95 \\
\hline 10 a 19 & 82 & 16.06 & 16.88 & 19.30 & 22.10 & 23.90 & 28.56 & 30.76 \\
\hline 20 a 29 & 64 & 16.78 & 19.26 & 21.70 & 23.90 & 25.95 & 29.36 & 42.95 \\
\hline 30 a 39 & 19 & 17.46 & 17.89 & 22.10 & 25.35 & 31.62 & 36.75 & 39.62 \\
\hline 40 a 49 & 29 & 16.92 & 18.10 & 20.07 & 23.40 & 26.80 & 29.60 & 33.65 \\
\hline 50 a 59 & 24 & 10.22 & 11.70 & 17.60 & 19.90 & 22.10 & 31.40 & 34.74 \\
\hline 60 a 69 & 10 & 15.00 & 15.22 & 18.62 & 19.45 & 20.87 & 35.32 & 36.80 \\
\hline
\end{tabular}

Fuente: Elaborado por los autores.

Tabla 7. Categorías de la fuerza de agarre en la mano dominante y no dominante para hombres

\begin{tabular}{|c|c|c|c|c|c|c|}
\hline \multicolumn{7}{|c|}{ Fombres } \\
\hline $\begin{array}{c}\text { Rangos de } \\
\text { edad }\end{array}$ & Deficiente & Mala & Regular & Bueno & Muy bueno & Excelente \\
\hline 10 a 19 & $<22.00$ & $22.01-28.70$ & $28.71-32.60$ & $32.61-43.65$ & $43.66-48.56$ & $>48.56$ \\
\hline 20 a 29 & $<30.24$ & $30.25-37.07$ & $37.08-40.20$ & $40.21-45.85$ & $45.86-48.86$ & $>48.86$ \\
\hline 30 a 39 & $<21.24$ & $21.25-28.90$ & $28.91-39.20$ & $39.21-48.70$ & $48.71-56.14$ & $>56.14$ \\
\hline
\end{tabular}




\begin{tabular}{|c|c|c|c|c|c|c|}
\hline 40 a 49 & $<25.52$ & $25.52-30.70$ & $30.71-39.00$ & $39.01-46.30$ & $46.31-49.36$ & $>49.36$ \\
\hline 50 a 59 & $<19.00$ & $19.01-25.50$ & $25.51-33.60$ & $33.61-37.05$ & $37.06-45.38$ & $>45.38$ \\
\hline 60 a 69 & $<21.10$ & $21.11-24.70$ & $24.71-30.10$ & $30.11-38.50$ & $38.50-41.74$ & $>41.74$ \\
\hline \multicolumn{7}{|c|}{ Fuerza de Mano no dominante (kg) } \\
\hline $\begin{array}{c}\text { Rangos de } \\
\text { edad }\end{array}$ & Deficiente & Mala & Regular & Bueno & Muy bueno & Excelente \\
\hline \begin{tabular}{c}
10 a 19 \\
\hline 20 a 29
\end{tabular} & $<21.48$ & $21.49-26.05$ & $26.06-32.80$ & $32.81-40.55$ & $40.56-49.20$ & $>49.20$ \\
\hline 30 a 39 & $<19.50$ & $19.51-31.80$ & $31.81-40.30$ & $40.31-47.25$ & $47.26-52.46$ & $>52.46$ \\
\hline 40 a 49 & $<18.22$ & $18.22-27.20$ & $27.21-38.50$ & $38.51-41.40$ & $41.41-46.44$ & $>46.44$ \\
\hline 50 a 59 & $<17.20$ & $17.21-27.30$ & $27.31-30.50$ & $30.51-39.25$ & $39.26-46.48$ & $>46.48$ \\
\hline 60 a 69 & $<17.54$ & $17.55-24.30$ & $24.31-27.20$ & $27.21-33.70$ & $33.71-35.70$ & $>35.70$ \\
\hline
\end{tabular}

Fuente: Elaborado por los autores.

Tabla 8. Categorías de la fuerza de agarre en la mano dominante y no dominante para mujeres

\begin{tabular}{|c|c|c|c|c|c|c|}
\hline \multicolumn{7}{|c|}{ Mujeres } \\
\hline \multicolumn{7}{|c|}{ Fuerza de Mano dominante (kg) } \\
\hline $\begin{array}{l}\text { Rangos de } \\
\text { edad }\end{array}$ & Deficiente & Mala & Regular & Bueno & Muy bueno & Excelente \\
\hline 10 a 19 & $<18.20$ & $18.21-19.90$ & $19.91-22.20$ & $22.21-25.30$ & 25.31-29.92 & $>29.92$ \\
\hline 20 a 29 & $<18.72$ & $18.73-22.60$ & $22.61-25.70$ & $25.71-28.35$ & $28.35-33.54$ & $>33.54$ \\
\hline 30 a 39 & $<19.49$ & $19.50-24.27$ & $24.28-28.45$ & $28.46-31.70$ & $31.70-39.33$ & $>39.33$ \\
\hline 40 a 49 & $<17.60$ & 17.61-18.92 & $18.93-23.45$ & $23.46-27.40$ & $27.41-32.65$ & $>32.65$ \\
\hline 50 a 59 & $<15.08$ & $15.08-18.70$ & $18.71-20.40$ & $20.41-22.70$ & $22.71-29.44$ & $>29.44$ \\
\hline 60 a 69 & $<11.53$ & $11.54-16.77$ & $16.78-19.30$ & $19.31-26.40$ & $26.41-42.33$ & $>42.33$ \\
\hline \multicolumn{7}{|c|}{ Fuerza de Mano no dominante (kg) } \\
\hline $\begin{array}{c}\text { Rangos de } \\
\text { edad }\end{array}$ & Deficiente & Mala & Regular & Bueno & Muy bueno & Excelente \\
\hline 10 a 19 & $<16.88$ & $16.88-19.30$ & $19.31-22.10$ & $22.11-23.90$ & $23.91-28.56$ & $>28.56$ \\
\hline
\end{tabular}




\begin{tabular}{|c|c|c|c|c|c|c|}
\hline 20 a 29 & $<19.26$ & $19.26-21.70$ & $21.71-23.90$ & $23.91-25.95$ & $25.96-29.36$ & $>29.36$ \\
\hline 30 a 39 & $<17.89$ & $17.89-22.10$ & $22.11-25.35$ & $25.36-31.62$ & $31.63-36.75$ & $>36.75$ \\
\hline 40 a 49 & $<18.10$ & $18.70-20.07$ & $20.08-23.40$ & $23.41-26.80$ & $26.81-29.60$ & $>29.60$ \\
\hline 50 a 59 & $<11.70$ & $11.70-17.60$ & $17.60-19.90$ & $19.91-22.10$ & $22.11-31.40$ & $>31.40$ \\
\hline 60 a 69 & $<15.22$ & $15.22-18.62$ & $18.62-19.45$ & $19.46-20.87$ & $20.88-35.32$ & $>35.32$ \\
\hline
\end{tabular}

Fuente: Elaborado por los autores.

(38-40). Por otra parte, en este estudio se manifestó que no existieron diferencias significativas entre la fuerza de agarre en la mano dominante y la mano no dominante en ninguna década para ambos sexos debido a que la fuerza en la mano dominante es aproximadamente un $10 \%$ más fuerte que la mano no dominante (41), encontrándose en otros estudios que el porcentaje de diferencia es menor al $10 \%$ tanto en hombres como en mujeres cuando la mano derecha es más fuerte que la izquierda o la izquierda más fuerte que la derecha (42-44), tal como se aprecia en nuestro estudio en el cual no hubo diferencias significativas en la mano dominante frente a la mano no dominante, probablemente por el hecho de que los sujetos de esta investigación estén en la necesidad de usar la mano no dominante durante sus actividades diarias (44).

Una investigación en Inglaterra concluyó que se debe tener cuidadoso cuando se vayan a hacer comparaciones entre el lado sano y el afectado, especialmente cuando se tiene como fin plantear una recuperación funcional del paciente para poder reincorporarse a sus actividades habituales (45). De otra parte, hay que tener en cuenta si se realiza actividad física en donde intervengan los miembros superiores o si ejercen algún tipo de labor para tener una mayor fuerza prensil.

La investigación presenta algunas limitaciones que pueden ser superadas en estudios posteriores, implementando otras metodologías y protocolos de medición, tal como la recolección de información antropométrica de extremidades superiores con la finalidad de identificar si existe asociación de la pérdida de fuerza prensil de mano por la masa muscular o masa grasa. Por otra parte, los datos obtenidos no se pueden generalizar para toda la población de la ciudad de San José de Cúcuta por el muestreo no probabilístico intencional empleado, que limita la muestra estudiada. Por último, se podría sugerir para próximas investigaciones a nivel internacional y nacional la ampliación de la muestra estudiada con un mayor control de las diferentes variables antropométricas, sociodemográficas y nutricionales, con el propósito de establecer valores de referencia que permitan determinar si un sujeto se encuentra en un estado óptimo o inadecuado con respecto a la década a la que pertenece.

\section{Conclusiones}

Este estudio permitió identificar que en una población, aparentemente sana, de la ciudad de Cúcuta los hombres entre los 10 y 69 años presentan valores más altos de fuerza de agarre en ambas manos con respecto a las mujeres en todas las décadas. Por otro lado, se evidenció que los valores medios más elevados de fuerza de agarre se obtuvieron en la tercera década, y a partir de la cuarta década empezaban a descender para ambos sexos y ambas manos.

Se recomienda que los baremos obtenidos a través de los percentiles sean empleados en el diagnóstico clínico para valorar la fuerza prensil de la mano en la población de la ciudad de Cúcuta y con ello categorizar la aptitud músculo-esquelética acorde al sexo y la edad.

\section{Conflicto de interés}

Los autores declaran no tener conflicto de interés.

\section{Agradecimientos}

Gracias a todas las personas que participaron voluntariamente y a la Universidad de Pamplona por apoyar este estudio.

\section{Referencias}

1. Mahn JK, Romero CP. Evaluación de la fuerza de puño en sujetos adultos sanos mayores de 20 
años de la región metropolitana [Tesis en Internet]. [Santiago de Chile]: Universidad de Chile; 2005 [citado 21 de diciembre de 2018]. Recuperado a partir de: http://www.repositorio.uchile.cl/handle/2250/110637

2. García DG, Piñera JA, García, A, Bueno C. Estudio de la fuerza de agarre en adultos mayores del municipio plaza de la revolución. Revista Cubana de Medicina Deportiva y Cultura Física. [Internet]. 2013 [citado 21 de diciembre de 2018];8(1):1-13. Recuperado a partir de: https://bit.ly/302Wid3

3. Poblete F, Flores C, Abad A, Díaz E. Funcionalidad, fuerza y calidad de vida en adultos mayores activos de Valdivia. Revista de Ciencias de la Actividad Física UCM [Internet]. 2015 [citado 21 de diciembre de 2018];16(1):45-52. Recuperado a partir de: http://revistacaf.ucm.cl/article/view/71/75

4. Rojas J, Vázquez L, Sánchez G, Banik S, Argáez J. Dinamometría de manos en estudiantes de Mérida, México. Rev. chil. nutr. 2012;39(3):45-51. doi: $10.4067 / \mathrm{S} 0717-75182012000300007$

5. Carreira C, Redondo C. Dinamometría manual y factores asociados en adolescentes. [Tesis de Máster en Internet]. [Cantabria]: Universidad de Cantabria; Cantabria; 2015 [citado 21 de diciembre de 2018]. Recuperado a partir de: http://hdl.handle. net $/ 10902 / 7673$

6. Vivas-Díaz AJ, Ramírez-Vélez R, Correa-Bautista JE, Izquierdo M. Valores de fuerza prensil por dinamometría manual en universitarios de Colombia. Nutr Hosp. 2016;33(3):330-336. doi: 10.20960/ nh. 113

7. Curcio CL, Gómez JF. Fuerza de agarre de los adultos mayores de los centros día del municipio de Manizales. Rev. Asoc. Colomb. Gerontol. Geriatr. [Internet]. 2005 [citado 21 de diciembre de 2018];19(4):849-858. Recuperado a partir de: http:// acgg.org.co/pdf/pdf revista 05/19-4-articulo1.pdf

8. Hincapié OL. Elaboración de estándares de la fuerza de agarre en individuos sanos entre 20 y 70 años residentes en la localidad de Usaquén, Bogotá. Rev. Colomb. Rehabil. 2007;6(1):5-20. doi: 10.30788/ RevColReh.v6.n1.2007.97

9. Ramírez PC, Angarita A. Fuerza de agarre en trabajadores sanos de Manizales. Rev. Colomb. Rehabil. 2009;8(1):109-118. doi: 10.30788/RevColReh. v8.n1.2009.171

10. Agredo V, Ramírez JD, Correa LJ, Montoya S, Idarraga $\mathrm{CA}$. Caracterización de la fuerza de agarre en la población entre 18 y 62 años de la ciudad de Medellín (Antioquia). 2009-2010 [Tesis en Internet]. [Medellín] Universidad CES; 2015 [citado 21 de diciembre de 2018] Recuperado a partir de: http:// hdl.handle.net/10946/784

11. Bustos-Viviescas BJ, Rodríguez-Acuña LE, Acevedo-Mindiola AA, Duran-Luna, LA, Lozano-Zapata RE. Influencia del porcentaje graso en la fuerza prensil de la mano en sujetos sanos de la ciudad de Cúcuta. En: III Encuentro de Investigación en Medicina y Salud. Universidad de Pamplona: Colombia. 2017a.

12. Bustos-Viviescas $\quad$ BJ, Rodríguez-Acuña LE, Acevedo-Mindiola AA, Ortì-Novoa JA, Duran-Luna, LA, Lozano-Zapata RE. Asociación entre la fuerza prensil de la mano y la presión arterial en sujetos aparentemente sano de la ciudad de Cúcuta. En: I Encuentro Interinstitucional de Semilleros de Investigación. Universidad Francisco de Paula Santander: Colombia. 2017 b.

13. Sánchez, AN, Bustos BJ, Acevedo AA. Los beneficios de la Actividad Física, como factor fundamental del buen estado físico y salud de las personas que asisten los días domingos a la ciclovía del malecón de la ciudad de Cúcuta. En: Universidad de Pamplona, editores. II Encuentro de Investigación en Medicina y Salud [Internet]. 2016 [citado 21 de diciembre de 2018]. P. 11-12. Recuperado a partir de: https://bit.ly/2VST2C1

14. Arias FG. El proyecto de investigación. Introducción a la metodología científica. Sexta edición. Caracas: Editorial Episteme; 2012. 146 p.

15. Declaración de Helsinki de la AMM-Principios éticos para las investigaciones médicas en seres humanos [Internet]. Instituto de Salud Carlos III. 2013. Recuperado a partir de: https://bit.ly/2q7WZDo

16. Harriss DJ, Atkinson G. Ethical standards in sport and exercise science research: 2014 update. Int J Sports Med. 2013;34(12):1025-1028. doi: 10.1055/ s-0033-1358756

17. Ministerio de Salud y Protección Social. Resolución Número 8430 de 1993. [Internet] Bogotá: Ministerio de Salud 1993. Recuperado a partir de: https://www.minsalud.gov.co/sites/rid/ Lists/BibliotecaDigital/RIDE/DE/DIJ/RESOLUCION-8430-DE-1993.PDF

18. Cossio-Bolaños MA, Arruda M. Propuesta de valores normativos para la evaluación de la aptitud física en niños de 6 a 12 años de Arequipa, Perú. Rev Med Hered. [Internet]. 2009 [citado 21 de diciembre de 2018];20(4):206-212. Recuperado a partir de: http://www.scielo.org.pe/scielo.php?script=sci arttext\&pid=S1018-130X2009000400006\&lng=es.

19. Rantanen T, Era P, Heikkinen E. Physical activity and the changes in maximal isometric strength in men and women from the age of 75 to 80 years. J Am Geriatr Soc. [Internet]. 1997 [citado 21 de 
diciembre de 2018];45(12):1439-1445. Recuperado a partir de: https://www.ncbi.nlm.nih.gov/ pubmed/9400552

20. Rantanen T, Guralnik JM, Foley D, Masaki K, Leveille S, Curb JD, White L. Midlife hand grip strength as a predictor of old age disability. JAMA. 1999a;281(6):558-560. doi: 10.1001/ jama.281.6.558

21. Rantanen T, Guralnik JM, Sakari-Rantala R, Leveille S, Simonsick EM, Ling S, Fried LP. Disability, physical activity, and muscle strength in older women: the women's health and aging study. Arch Phys Med Rehabil. [Internet]. 1999b [citado 21 de diciembre de 2018];80(2):130-135. Recuperado a partir de: https://www.ncbi.nlm.nih.gov/pubmed/10025485

22. Reed RL, Pearlmutter L, Yochun K, Meredith KE, Mooradian AD. The relationship between muscle mass and strength in the elderly. J Am Geriatr Soc. [Internet]. 1991 [citado 21 de diciembre de 2018];39(6):555-561. Recuperado a partir de: https://www.ncbi.nlm.nih.gov/pubmed/1805811

23. Reed RL, Den Hartog R, Yochum K, Pearlmoner L, Ruttinger CA, Mooradian DA. A comparison of hand held isometric strength measurement with isolament muscle strength measurement in the elderly. J Am Geriatr Soc. 1993;41(1):53-55. doi: 10.1111/j.1532-5415.1993.tb05949.x

24. Laukkanen P, Era P, Heikkinen RL, Suutama T, Kauppinen E, Heikkinen E. Factors related to carrying out everyday activities among elderly people aged 80. Aging [Internet]. 1994 [citado 21 de diciembre de 2018];6(6):433-443. Recuperado a partir de: https://www.ncbi.nlm.nih.gov/pubmed/7748917

25. Luna-Heredia E, Martín-Peña G, Ruiz J. Valores normales y límites de la normalidad de la fuerza de la mano determinados con dinamometría. Nutr. Hosp. [Internet]. 2004 [citado 21 de diciembre de 2018];19(supl.1):80. Recuperado a partir de: http://scielo.isciii.es/scielo.php?script=sci arttext\&pid $=$ S0212-16112004000700074

26. Henneberg M, Brush C, Harrison A. Growth of specific muscle strength between 8 and 18 years in contrasting socioeconomic conditions. Am. J. Phys. Anthropol. 2001;115(1):62-70. doi: 10.1002/ajpa.1057

27. Malina RM, Little BB. Physical Activity: The Present in the Context of the Past. Am. J. Hum. Biol. 2008;20(4):373-391. doi: 10.1002/ajhb.20772

28. Massy-Westropp N, Gill T, Taylor A, Bohannon R, Hill C. Hand Grip Strength: Age and gender stratified normative data in a population-based study. BMC Research Notes. 2011;4:127-131. doi: 10.1186/1756-0500-4-127

29. Barbosa JAP, Rodríguez M., Hernández YM,
Hernández RA, Herrera HA. Masa muscular, fuerza muscular y otros componentes de funcionalidad en adultos mayores institucionalizados de la Gran Caracas-Venezuela. Nutr.Hosp. [Internet]. 2007 [citado 21 de diciembre de 2018];22(5):578-583. Recuperado

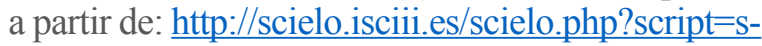
ci arttext\&pid=S0212-16112007000700009

30. Oteo JA, Benavente P, Garzón M. Valores normativos de la fuerza de puño en la población española en edad laboral. Influencia de las variables antropométricas de la mano y el antebrazo. Rev Iberoamericana de Cirugía de la Mano. 2015;43(2):104-110. doi: 10.1016/j.ricma.2015.09.005

31. Schlüssel MM, dos Anjos LA, de Vasconcellos MT, Kac G. Reference values of handgrip dynamometry of healthy adults: a population-based study. Clinical Nutrition. 2008;27(4):601-607. doi: 10.1016/j. clnu.2008.04.004

32. Daly R, Rosengren B, Alwis G, Ahlborg H, Sernbo I, Karlsson M. Gender specific age-related changes in bone density, muscle strength and functional performance in the elderly: a-10 year prospective population-based study. BMC Geriatrics. 2013;13:71-79. doi: 10.1186/1471-2318-13-71

33. Stessman J, Rottenberg Y, Fischer M, Hammerman-Rozenberg A, Jacobs JM. Handgrip Strength in Old and Very Old Adults: Mood, Cognition, Function, and Mortality. J Am Geriatr Soc. 2017;65(3):526-532. doi: 10.1111/jgs.14509

34. Baumgartner RN, Koehler KM, Gallagher D, Romero L, Heymsfield SB, Ross RR, Garry PJ, Lindeman RD. Epidemiology of sarcopenia among the elderly in New Mexico. American J Epidemiology. 1998;147(8):755-763. doi: 10.1093/oxfordjournals. aje.a009520

35. Janssen I, Shepard DS, Katzmarzyk PT. The healthcare costs of sarcopenia in the United States. J Am Geriatr Soc. 2004;52(1):80-85. doi: 10.1111/j.15325415.2004.52014.x

36. Luna-Heredia E, Martín-Pena G, Ruiz-Galiana J. Handgrip dynamometry in healthy adults. Clinical Nutrition. 2005;24(2):250-258. doi: 10.1016/j. clnu.2004.10.007

37. Izquierdo M. Biomécanica y bases neuromusculares de la actividad física y el deporte. Madrid: Editorial Médica Panamericana; 2008. 784 p.

38. Torres M, González del Pino J, Yáñez J, Bartolomé del Valle E. Estudio dinamométrico de la mano y el pulgar. Rev Esp Cirugía Ortopédica y Traumatología [Internet]. 1999 [citado 21 de diciembre de 2018];43(5):321-326. Recuperado a partir de: http:// www.elsevier.es/es-revista-revista-espanola-cirugia-ortopedica-traumatologia-129-articulo-estu- 
dio-dinamometrico-mano-el-pulgar-13007234

39. Hanten WP, Chen WY, Austin AA, Brooks RE, Carter HC, Law CA, Morgan MK, Sanders DJ, Swan CA, Vanderslice AL. Maximun grip strenght in normal subjects from 20 to 64 years of age. J Hand Ther. [Internet]. 1999 [citado 21 de diciembre de 2018];12(3):193-200. Recuperado a partir de: https://www.ncbi.nlm.nih.gov/pubmed/10459527

40. Kamarul T, Ahmad TS, Loh WY. Hand grip strength in the adult Malaysian population. J Orthop Surg. 2006;14(2):172-177. doi: $10.1177 / 230949900601400213$

41. Incel NA, Ceceli E, Durukan PB, Erdem HR, Yorgancioglu ZR. Grip strength: effect of hand dominance. Singapore Med J. [Internet]. 2002 [citado 21 de diciembre de 2018];43(5):234-237. Recuperado a partir de: https://www.ncbi.nlm.nih.gov/ pubmed/12188074
42. Lorenzo-Agudo MA, Santos-García P, Sánchez-Belizón. Determinación de los valores normales de fuerza muscular de puño y pinza en una población laboral. Rehabilitación. 2007;41(5):220-227. doi: 10.1016/S0048-7120(07)75521-4

43. Pamela DA, Jeannette O, Verónica S, Fresia F. Parámetros de Normalidad en Fuerzas de Prensión de Mano en Sujetos de Ambos Sexos de 7 a 17 Años de Edad. Rev Chl Ped. 2009;80(5):435-443. doi: 10.4067/S0370-41062009000500005

44. Rojas JA, Vázquez LC, Sánchez GV, Banik SD, Argáez J. Dinamometría de manos en estudiantes de Mérida, México. Rev Chl Nutr. 2012;39(3):45-51. doi: 10.4067/S0717-75182012000300007

45. Armstrong CA, Oldham JA. A comparison of dominant and non-dominant hand strengths. J Hand Surg (Edinburgh, Scotland). 1999;24(4):421-425. doi: 10.1054/jhsb.1999.0236 